\title{
Concepciones de los docentes de básica primaria sobre educación inclusiva en la institución educativa María inmaculada de San Benito Abad Sucre - Colombia
}

\author{
Julia Del Rosario Madero Chamorro \\ Universidad de Sucre, Colombia \\ megustasbio@gmail.com
}

\begin{abstract}
Resumen
Esta es una investigación cualitativa cuyo objetivo fue conocer las concepciones sobre la educación inclusiva que tienen los docentes de básica primaria de la Institución Educativa María Inmaculada de San Benito Abad - Sucre, Colombia. Se realizó mediante el paradigma hermenéutico que interpreta la realidad de los docentes en un contexto particular, evitando generar interferencia. Se llevó a cabo un estudio de caso único. La información se recolectó a través de entrevistas semiestructuradas, observaciones no participantes, de cinco docentes de la Institución, para triangular su pensamiento y conocer la concepción de sus saberes sociales cristalizados en discursos que han sido construidos por la práctica directa y no mediada por los sujetos protagonistas de la acción. La importancia del presente estudio se evidencia en que con ella se puede propiciar la reflexión del quehacer pedagógico de los docentes, en cuanto al tema de la inclusión. Además, permite a partir de los resultados se genere otros estudios enfocados al tema.
\end{abstract}

Palabras clave: Educación Inclusiva, Diversidad, Docentes y Educación Básica Primaria.

\section{Conceptions of primary school teachers on inclusive education at the María Inmaculada school in San Benito Abad Sucre - Colombia}

\begin{abstract}
This is a qualitative research whose objective was to know the conceptions about inclusive education that primary school teachers have on the María Inmaculada Educational Institution of San Benito Abad - Sucre, Colombia. It was made through the hermeneutic paradigm that interprets the reality of teachers in a particular context, avoiding generating interference. A single case study was carried out. The information was collected through semi-structured interviews, non-participant observations, of five teachers of the Institution, to triangulate their thinking and know the conception of their social knowledge crystallized in discourses that have been constructed by direct practice and not mediated by the subjects protagonists of the action. The importance of the present study is evident in that it is possible to foster reflection on the pedagogical work of teachers, in terms of inclusion. In addition, it allows other studies based on the subject to be generated from the results.
\end{abstract}

Key Words: Inclusive Education, Diversity, Teachers and Basic Primary Education. 


\section{Introducción}

En las últimas décadas han sido muchas y diversas las iniciativas que se han llevado a cabo con objeto de transformar nuestras aulas y centros en entornos de aprendizaje y de desarrollo de los estudiantes, y en particular, de aquellos con mayor riesgo de exclusión. Desde los programas de integración, que en los años setenta y ochenta tenían como objetivo principal que los estudiantes con algún tipo de barreras para el aprendizaje pudieran ser atendidos en los centros ordinarios, hasta el progresivo convencimiento de que la mera integración escolar resultaba un objetivo insuficiente, y a menudo equívoco. Durán. D; y Giné. C. (2010).

Es así, cómo la inclusión remueve los planteamientos más profundos de una auténtica educación: destaca el derecho fundamental de todos a recibir una educación de calidad, incorpora la realidad humana de la diversidad como un valor, plantea el medio ordinario como el más realista, natural y eficaz para llevar a cabo dicha educación, exige la participación y convivencia como metas integrantes de todo proceso educativo, demanda el desarrollo de un currículo funcional, común y adaptado a la vez a la individualidad de cada estudiante, promueve un aprendizaje significativo, cooperativo, constructivista, reflexivo. Finalmente, involucra a toda la comunidad educativa y a la sociedad misma como marcos y agentes de la educación. López, M. (2009).

Estainvestigaciónnace, porlamismanecesidad de iniciar un proceso de transformación de las aulas e instituciones en entornos de aprendizaje y de desarrollo de todos los estudiantes, y en particular de aquellos con mayor riesgo de exclusión, para así gestar una propuesta educativa que, puesta en práctica en estos contextos, pudiera dar luz y facilitar el tránsito progresivo hacia lo inclusivo que conozca, que comprenda y que respete la diversidad de los estudiantes en las aulas. Desde esta perspectiva, muy posiblemente las prácticas educativas se convierten en un proceso de comunicación humana, con la finalidad de abrir espacios de cooperación entre todos los estudiantes.
Teniendo en cuenta lo anterior y observando desde nuestra labor docente, que algunos elementos del proceso educativo de la Institución Educativa María Inmaculada de San Benito Abad Departamento de Sucre Colombia, no responden a las particularidades de la población escolar, ya que no se ha podido superar límites como la supuesta homogenización de los estudiantes y de los procesos de aprendizaje, para el cual el sistema educativo mantiene el "statu quo" y son los estudiantes quienes se tienen que adaptar a la escuela y no ésta a los estudiantes.

Es así que el objetivo de la presente investigación es comprender las concepciones que tienen los docentes de básica primaria de la Institución Educativa María Inmaculada de San Benito Abad Sucre sobre la educación inclusiva; abordando primordialmente las demandas sociales del profesorado y la imagen que este tiene sobre la educación inclusiva y su función de educadores teniendo en cuenta sus consideraciones, sensibilidades y flexibilidades.

\section{Metodología}

La metodología se fundamentó en el enfoque cualitativo, condiseñodescriptivo-interpretativo. El estudio se orientó a investigar la compleja realidad social, por lo que en el transcurso de la recogida de datos, se fueron acumulando variados textos y contextos provenientes de diferentes técnicas. La interpretación se analizó de manera sistemática, encaminada a generar constructos y establecer relaciones entre las variables intervinientes. Constituyéndose esta metodología, en un camino para llegar de modo coherente a la construcción de conocimiento.

El método adoptado para la investigación fue el estudio de caso, ya que resultó apropiado para abordar la diversidad y complejidad de los actores sujeto de estudio, según McMillan \& Schumacher (2005), este método facilita el entendimiento de un concepto abstracto a partir de la experiencia de los participantes. Relaciona e integra percepciones sobre un determinado hecho para sugerir y construir propuestas de abordaje del fenómeno estudiado. 
Además, para Rodríguez, G. Gil, J \& García, E, (1996) el estudio de caso también contribuye al desarrollo y transformación de prácticas cotidianas. Bien sea proporcionando un análisis detallado de la dinámica de trabajo particular y del proceso, o para aumentar los niveles de comprensión y mejora de dicha práctica. Así mismo este tipo de estudio es significativo al tener sustento en la replicación, entendida ésta como la capacidad que tiene de analizar y contrastar las respuestas que se obtienen de forma parcial con cada caso estudiado.

De acuerdo a la clasificación realizada por Stake (2007) en esta investigación el tipo de estudio de caso seleccionado fue caso único, porque se estudió o se indagó un fenómeno como es la Educación inclusiva, donde el interés se centró, no en un caso, sino, en un número representativo de docentes que tienen niños con alguna discapacidad incluidos en su aula. Para el presente estudio se focalizó a docentes de básica primaria de la Institución Educativa María Inmaculada, centrando el interés en conocer y comprender las concepciones de estos sobre la Educación inclusiva.

Igualmente es descriptiva porque describió las concepciones de los sujetos involucrados en el estudio. No pretendió comprobar o verificar hipótesis, sino que, a partir de categorías definidas con anterioridad, se trató de descubrir situaciones referidas a las concepciones sobre Educación Inclusiva de los docentes de básica primaria. En este aspecto cabe señalar la definición de investigación descriptiva dada por Tamayo, M (2002) “Comprende la descripción, registro, análisis e interpretación de la naturaleza actual, o sobre como una persona, grupo o cosa se conduce o funciona en el presente" (p. 46).

Este tipo de investigación permitió conocer directamente a los investigados (Docentes) sus puntos de vista, concepciones, creencias, interpretaciones, significados sobre el proceso de educación inclusiva que se realizó en la Institución Educativa María Inmaculada. A partir de allí, el conocimiento generado se trianguló con elementos teóricos, producto de la investigación científica y de este modo, generar un nuevo conocimiento que transforme las concepciones, percepciones y prácticas de la comunidad docente sobre la educación Inclusiva.

La población, sujeto de estudio, está conformada por 5 docentes, los cuales orientan las áreas de castellano, educación física, matemáticas, inglés, informática, artística, ciencias naturales, religión, ética y valores, en los grados $1^{\circ}$ a $5^{\circ}$ de básica primaria, sin rotación de grados.

En cuanto al grupo de apoyo es preciso señalar que esté se tomó teniendo en cuenta la intención de los investigadores, dado que se quiere comprender las concepciones que tienen los docentes de la Institución Educativa María Inmaculada de San Benito Abad Sucre sobre la educación inclusiva. La información requerida encontró representatividad en los docentes de básica primaria. El grupo colaborador está conformado por 5 docentes, fue tomada de manera intencional, evitando el sesgo y la subjetividad.

Para la selección de ésta se tendrá en cuenta criterios como: experiencia del docente y la inclusión de niños con algún tipo de necesidad especial en su grupo de trabajo.

La información que posteriormente se analizó, fue recolectada mediante diferentes técnicas e instrumentos, los cuales fueron seleccionados teniendo en cuenta la naturaleza cualitativa de esta investigación, ellas son: Observaciones directas y Entrevistas.

En primera instancia se optó por la técnica de observación, dado que a través de ésta se pueden registrar las principales características que representan la conducta de las personas frente a un determinado fenómeno y los sucesos que se generan a partir de dicha conducta, o del fenómeno en sí mismo, como afirma Virginia Aragón Jiménez (2010):

Para obtener una información general del funcionamiento y de la actuación de los individuos en un ambiente determinado, la observación se convierte en el instrumento 
esencial que nos permite registrar y asignar un significado a lo percibido de acuerdo con el contexto en el que nos encontramos. Dentro del ámbito educativo, la primera toma de contacto con el Docente concreto se realiza a través de la observación. (p. 1)

Se realizaron observaciones a los docentes que orientan todas las áreas, en el nivel de básica primaria de la Institución Educativa María Inmaculada. El propósito de dichas observaciones fue identificar cómo los docentes involucran la educación inclusiva en sus clases, qué estrategias y criterios tienen en cuenta para incluir a los estudiantes con algún tipo de necesidad educativa durante las clases.

Para llevarlas a cabo se elaboró un protocolo el cual está enmarcado dentro de las categorías que forman parte de los fundamentos teóricos de esta investigación (Educación inclusiva; relaciones, concepción y diversidad). Este protocolo se toma como base para desarrollar la técnica de la observación, no obstante, pueden ampliarse dependiendo de lo que surja o no en el evento de clase.

Por todo lo descrito aquí, es necesario señalar que el tipo de observación que se realizó es directa y no participante, "dado que los investigadores pudieron observar mediante su propia observación, sin ocupar un nivel o función dentro del aula de clases". Tamayo, M (2002, p.183). Se definieron claramente los comportamientos a observar e igualmente, se identificó el problema que dio origen a esta investigación $y$, por consiguiente, la información que se necesita recolectar está especificada.

Las observaciones se realizaron durante un periodo de un semestre, aplicándose el mismo protocolo para cada docente, con una duración de 20 minutos cada una, en diferentes oportunidades. Es de resaltar que, aunque el protocolo fue diseñado de acuerdo a los objetivos, en el transcurso de las observaciones emergieron datos relevantes para la investigación.

Otra de las técnicas utilizadas fue la entrevista que se describe como "...encuentros dirigidos hacia la comprensión de la perspectivas que tienen los entrevistados respecto de sus vidas, experiencias o situaciones" Taylor y Bogdan (1990, p. 101). Aunque existen numerosas clasificaciones, se ha optado por la que establece Ruiz, J. (1999) para delimitar la empleada en este estudio, según los parámetros que establece este autor: individual vs grupal, enfocada vs de amplio espectro y dirigidas vs no estructuradas o semiestructurada. Según esta clasificación, se opta por la entrevista individual, y semiestructurada ya que es la que más se ajusta a los objetivos planteados. Además, ayudará a recoger la información necesaria para responder al interrogante generado en el problema, por ello se realizaron entrevistas individuales.

En lo relacionado con la entrevista semi estructurada, se diseñó un guion de acuerdo al propósito que se pretendió abordar, el cual se utilizó para entrevistar a los docentes. Esta entrevista se identifica con la sigla EDI, que quiere decir, entrevista a docentes de la investigación. A través de ella se pudo generar un diálogo fluido con preguntas abiertas y cerradas, estructuradas en un formato de estímulos para provocar respuestas ricas por parte de los docentes objeto de estudio. Se realizó primero una prueba piloto a los docentes involucrados para establecer la comprensión de las preguntas, y a medida que se avanzaba en la misma se iba retroalimentando con preguntas para mejorar la comprensión, sin olvidar los objetivos de la misma.

Realizada la recolección de la información a través de la aplicación de las observaciones realizadas a las prácticas de los docentes al interior del aula, y la aplicación de entrevistas se procedió a la descripción e interpretación de la misma.

\section{Resultados}

Las "Concepciones de Educación inclusiva" en la experiencia de los docentes, como orientadores de estudiantes que presentan barreras para el aprendizaje muestran que han tenido la oportunidad de conocerlos $\mathrm{y}$ han sido o son parte de su formación, aunque esta experiencia en la mayoría de los casos no tuvo un impacto 
positivo o gratificante. Los docentes D1, D2, D3, D4, y D5 expresaron: "que han sido pocas las experiencias que han tenido, sin embargo, en sus aulas hay integración de niños con alguna barrera de aprendizaje. Pero que no se le ha creado una temática que le permita asimilar de forma coherente y adaptativa". El docente D3 “Que era un reto recibirlo, por las estrategias de enseñanza". El docente 1 "Al tenerlos en mi grupo me dio mucha preocupación ya que no contaba con un equipo de apoyo interdisciplinario que me colaborara con el proceso de aprendizaje de estos".

No obstante, es necesario aclarar que esta tendencia desfavorable hacia la inclusión no es sinónimo de fracaso, como en algunos casos lo han dado a entender los docentes, por el contrario, estos resultados los podemos tomar como una posición a un cambio de actitud y mentalidad para fortalecer el proceso de inclusión.

De acuerdo a estas concepciones se deduce claramente que el profesorado inconscientemente está actuando bajo los parámetros de la homogenización y que para atender y brindarles una educación de calidad es necesario tener herramientas metodológicas especiales para dicho manejo, ya que según ellos no cuentan con la preparación adecuada para su atención integral, por tanto se evidencia en sus respuestas actitudes de exclusión, por lo que se puede concluir que generalmente la muestra presenta una tendencia actitudinal adversa hacia la inclusión de los estudiantes que presentan barreras de aprendizaje en la educación básica primaria de la Institución Educativa María Inmaculada.

Es así que "Una educación inclusiva”, necesita resignificar el espacio de la crítica desde la cultura y la historia. La cultura en esta perspectiva"... no se ve como monolítica o inmutable, sino como un lugar de fronteras múltiples y heterogéneas donde las diferentes historias, lenguajes, experiencias y voces se entremezclan en medio de diversas relaciones de poder y privilegio. Vega, A, (2005).

Dentro de esta tierra fronteriza cultural y pedagógica, conocida como escuela, las culturas subordinadas se empujan y penetran las supuestamente homogéneas y no problemáticas fronteras de las prácticas y formas culturales dominantes" Giroux, H (1992, p. 8).

La forma como los docentes conceptualizan las ideas sobre las diferencias de los estudiantes, los acerca preferentemente a un modelo de integración que a uno de inclusión. Por ejemplo, en un grupo numeroso de docentes el concepto de inclusión se reduce a la atención de personas discapacitadas, adoptando iniciativas de integración con prácticas procedentes de la educación especial, tales posturas provocan en institución educativa nuevas formas de segregación. Lo anterior se puede constatar en afirmaciones como las expuestas por el docente 1 al afirmar que "Me ha ayudado a cerrar brechas en mis propios logros ya que el aceptar el reto de escolarizar a niños cuyos aportes se ven a pasos lentos pero que le dan la seguridad y las herramientas para confrontar adversidades en su largo trasegar".

Es una etapa de profundas transformaciones en la educación debido a los cambios sociales, somos conscientes que se presentan nuevos enfoques educativos, problemas en la toma de decisiones en los objetivos propuestos, en las metodologías que se emplean, en fin, la educación ha necesitado y necesita plantearse y replantearse su discurso, por cuanto, se concibe la necesidad que el educador tome una actitud reflexiva de la problemática educativa de nuestro tiempo.

“La formación del profesorado se constituye en uno de los principales factores dinamizadores y cualificadores de los sistemas educativos. Se dice a menudo que los docentes solos no podrán cambiar la educación, pero si no cambian los docentes, difícilmente podrán lograrse transformaciones en ella. Los diferentes diagnósticos acerca de los mejores aprendizajes logrados por los estudiantes, y el incremento de la calidad de la educación, subrayan la importancia de los maestros como actores centrales en el proceso educativo" González, M. (2008, p. 11). 
El no tomar decididamente el compromiso de cambiar, causa dificultad en el momento que se evidencia el alcance de los objetivos de la educación inclusiva. Aunque, las guías a seguir por el MEN se destacan por su meritorio contenido, la experiencia nos muestra que todavía hay mucho por hacer para reducir las desigualdades y obrar con equidad: hay carencias básicas de infraestructura en colegios y escuelas, utilización de términos ofensivos hacia estudiantes con barreras para el aprendizaje (anormales, mongolitos, enfermitos, y otros estigmáticos más), pocas o nulas capacitaciones a docentes y administrativos sobre el tema, currículos y evaluaciones poco flexibles.

Esta demanda de modernidad se revela, ocasionando deserción y repitencia por parte de los estudiantes, sentimientos de impotencia, ira y aflicción por parte de sus padres, así como también inconformismo por parte de algunos profesores. Pero no es el hecho de criticar simplemente, hay que proponer, y no basta con reconocerlo, hay que actuar, aunque esta posición implique un sendero de tristes recaídas, pero también de muchísimos avances positivos colmados de satisfacciones.

Pero, como la realidad es una construcción del sujeto, y en esta realidad nos enfrentamos a la insensibilidad, miedos, facilismo e individualismo; todo lo que genere cambio o transformación causa inseguridad y ansiedad, asociadas a menudo a prejuicios e ideas que, trasmitidas de boca en boca, pueden dar una imagen confusa de la educación Inclusiva, haciendo que los procesos de atención a la diversidad se convierta en un riesgo para atender la educación para todos con calidad.

Cabe anotar que como seres humanos con principios y valores, hemos ido perdiendo el valor de la empatía, la responsabilidad y la ética; cualidadesnecesariasparaatender, guiaruorientar a nuestros estudiantes, más aún a estudiantes que requieren de nuestro profesionalismo y de pensamiento abierto hacia el cambio para que hagan historia y de alguna manera, de acuerdo a sus posibilidades contribuyan al desarrollo de nuestro país. Como lo afirma Blanco, R. (2002) quien dice que por cada niño que escolaricemos en estas condiciones contribuimos a formar una familia más prospera.

Es necesario tener presente que en nuestro sistema educativo se vive un proceso muy complejo, que implica cambios radicales en el ámbito actitudinal, conceptual, metodológico y organizacional con la finalidad de implementar o establecer otras normas o modelos de intervención en las instituciones educativas en los niveles de preescolar, básica y media, para que tengan un perfil que favorezca la inclusión de personas con barreras de aprendizaje. La dificultad de este proceso, entre otras ya mencionadas, está en la consolidación principalmente de los conceptos básicos que han de permitir la sensibilización, concientización y comprensión de la comunidad educativa y de la sociedad en general, con base en el principio de una educación para la diversidad.

Partiendodela cotidianidad delos estudiantes que de forma permanente y continua asisten a un establecimiento educativo, es oportuno volver la mirada hacia la preparación del docente quién es uno de los responsables de la educación del ciudadano. Además de impartir sus saberes en diferentes disciplinas (matemáticas, español, ciencias naturales, sociales, inglés, informática, entre otras) debe buscar respuestas que contribuyan a cristalizar sociedades más justas. No se trata de formar un docente poseedor de gran cantidad de conocimientos y transmisor de información, cuyo objetivo en la mayoría de los casos es alcanzar a cumplir con su programa de instrucción durante el año lectivo sino de propiciar el desarrollo de capacidades, actitudes $\mathrm{y}$ aptitudes para el aprendizaje permanente.

Estamos viviendo en una época muy acelerada, en donde se tiene que responder a sus exigencias acuciantes y prioritarias, por lo cual no se puede dejar de lado la importancia de saberes de todas las áreas que nos ofrece el sistema educativo, sin embargo es preciso detectar lo que no muestran nuestros estudiantes, lo invisible; es ver en ellos sus potencialidades para explotarlas y empezarlos a orientar para que lo utilicen en el beneficio y desarrollo de la humanidad. 
Pero ¿por qué este tecnicismo moderno es aplicado a los docentes, si ellos son seres humanos transformadores positivos de vida? Pues bien, para explicarlo se tomaron como muestra 2 preguntas realizadas a diferentes docentes: D1. ¿Cree que esta situación de atender niños/as con barreras para el aprendizaje enriqueció su experiencia de vida?

D.1. "Totalmente porque siente uno que puede superar metas.... Siento que con algunas estrategias se puede brindar un poco de aceptación social a la formación de estos niños.

D.3. "Si me enriqueció, porque en esta experiencia aprendí estrategias nuevas de aprendizaje. Por ejemplo, a través de la visión le indicaba cuando y en que momento necesitaba que ella transcribiera que ya era hora de recoger el material de trabajo. Mientras que D4 y D5 indican que "Si. Individualmente estar frente a estas situaciones te hace una persona sensible, solidaria y agradecida con Dios por su sabiduría para tratar estos casos.

Sí el docente tan sólo se diera la oportunidad de interactuar con un niño, niña o joven en condición de discapacidad, sin que fuera una camisa de fuerza obligada por el estado, sino que por el contrario lo recibiera como un reto positivo para transformar vidas, muy seguramente conocería la grandeza de la misión de educar y la magnitud de la responsabilidad social de la misma. Ciertamente, los docentes requieren ser conscientes que la educación Inclusiva traeel sello de compromiso, responsabilidad y amor, como elementos para anticipar respuestas a nuevos desafíos y generar alternativas de solución, pues las innovaciones educativas se presentan como un espacio crucial para la educación en y desde la diversidad.

Como se puede corroborar, el hecho de que los docentes compartan durante toda una jornada escolar con estudiantes que presenta barreras para el aprendizaje, es ya para ellos, sinónimo de incomodidad, ya sea por sus imaginarios, la prevención dada por experiencias de colegas, impotencia al brindarle una orientación significativa, falta de actualización profesional, cansancio por tener más de 25 años laborando o simplemente porque se requiere más compromiso, trabajo, tiempo y sobretodo tolerancia. Todas las anteriores características, fue posible concebirlas desde una sola categoría que llamamos "concepciones de la inclusión educativa".

\section{Conclusiones}

Con el presente trabajo se buscó "Comprender las concepciones que tienen los docentes de la Institución Educativa María Inmaculada de San Benito Abad Sucre sobre la educación inclusiva"

Es así, como a lo largo de ese análisis realizado a la información recolectada, se encontró una serie de percepciones, similitudes y contrastes relacionados con los diferentes aspectos que forman parte del proceso de enseñanza - aprendizaje. De todos esos hallazgos, se estableció a manera de síntesis las siguientes conclusiones.

Cada caso ha sido influenciado históricamente por un contexto sociocultural, el cual contribuye a establecer y construir determinadas concepciones en torno al tema de la educación inclusiva, el cual es visto desde diferentes perspectivas de acuerdo al momento y a las situaciones dadas.

Con la presente investigación se intentó propiciar espacios de interacción y por qué no de reflexión entre uno de los actores del proceso educativo, en uno de los temas más señalados y más juzgados en los últimos tiempos. Quien más, sino los involucrados directamente en este proceso nos pueden dar luces, para entender todo lo profundo y dinámico que encierra el tema de inclusión.

Las expresiones de los docentes a lo largo de este recorrido investigativo, reflejan unas concepciones que en algunas ocasiones carecen de claridad conceptual, lo que puede incidir en ellos al momento de reflexionar sobre el tema de la educación inclusiva, llegando a confundir el término de inclusión con el de diversidad. Esto se evidenció en las respuestas dadas en las 
entrevistas cuando solo se refieren al término de diferencias cuando se trata de niños con barreras para el aprendizaje, o evidente minusvalía. Así mismo, no relacionan la situación de extra edad con inclusión, sino por el contrario con una población de estudiantes en iguales condiciones. Al hacer el contraste con la práctica, cada uno de ellos desarrollan estrategias pedagógicas homogéneas, sin atender a la heterogeneidad de los grupos; por ejemplo, el caso D1 cuando se observó la clase y este ingresa al aula con un saludo en voz baja que no cobija la totalidad del grupo, Inicia la clase con el llamado a lista de los cuarenta integrantes del grupo e inicia la clase escribiendo un texto en el tablero, para después ser leído por los estudiantes. En este grupo previamente es conocido que hay tres estudiantes con barreras para el aprendizaje. Pero el ejercicio lector es para todos, sin diferenciación alguna.

En cuanto a las concepciones de educación inclusiva, los docentes tienen unas construcciones mentales referidas solo a estudiantes con problemas de aprendizaje, esto se refleja en las respuestas dada por D1, cuando se le pregunta por ¿que entiendes por inclusión?: "Es permitir en el aula regular niños con barreras cognitivas y físicas. Es aceptar, tener en cuenta la participación de todo ser humano", la anterior afirmación puede ser producto del desconocimiento de la normatividad que rige el sistema educativo en cuanto a políticas de inclusión, las cuales se quedan en el discurso teórico, pero no se refleja en su quehacer pedagógico, posiblemente por que la Institución Educativa a pesar que conocer la política, no desarrolla mecanismos que procuren su respectiva implementación.

Al realizar las observaciones también se percibe que el discurso no es coherente con las respuestas dadas en las entrevistas, porque, a pesar que reconoce que la inclusión tiene que ver con barreras de aprendizaje, al desarrollar la clase no tiene en cuenta a aquellos estudiantes que presentan dichas barreras, como la sordera de una niña que esta integrada al grupo. A medida que avanza la clase, D1 no hace contacto visual con la estudiante, no revisa los apuntes de la estudiante, y menos las actividades propuestas para realizar en casa.
Esta misma situación se observa en el caso de D2, cuando al desarrollar la clase no tiene en cuenta la presencia de un estudiante con síndrome de Asperger, ignorando todo lo que acontece alrededor de este caso, es decir, el estudiante no participa en la clase, se aleja del aula, juega solo, a lo que D2 no hace ninguna objeción, ni procura involucrar al niño en las actividades desarrolladas.

Al observar las relaciones establecidas entre los docentes con los estudiantes incluidos en los diferentes grupos, y en cuyas aulas se realizaron las observaciones se denoto poco acercamiento y comunicación entre estos, tanto así los D1 y D3 ignoran a sus estudiantes con barreras, llegando al punto de ubicarlos en la parte posterior del aula de clases, estratégicamente según ellos para evitar que distraigan al resto del grupo de los estudiante; consecuente a lo anterior los estudiantes muestran timidez al intentar establecer un dialogo con su profesor, es decir, la relación docente estudiante y viceversa carece de empatía afectando la ideal comunicación asertiva entre ellos, factor determinante para enriquecer la relación planteada anteriormente . Se produce entonces un aislamiento en lo que debería ser una relación de amistad mediada por el respeto y la confianza, que ayuden a minimizar las barreras para el aprendizaje que tanto daño le hacen al proceso mismo.

En general los profesores evidencian en sus prácticas pedagógicas actitudes positivas $\mathrm{y}$ negativas, que oscilan entre la indiferencia, sobreprotección, bajas expectativas y aceptación hacia los niños con necesidades educativas especiales. Las actitudes negativas o de indiferencia se ven reflejadas, por una parte, en la ubicación del alumno integrado al final de la sala de clases, impidiendo tener mayor contacto con el educador y por consiguiente dificultando la comprensión de las instrucciones y contenidos. De igual forma se evidencia falta de preocupación por hacer partícipes a los niños/ as con necesidades educativas especiales de las actividades planificadas para el grupo, a pesar de ciertos argumentos de algunos profesores que manifiestan hacer adaptaciones curriculares. En este sentido se refleja una clara disonancia entre la 
actuación pedagógica del profesor y su discurso. Las formas de relación que establecen algunos profesores de aula con los alumnos integrados en su acción pedagógica, dan cuenta de una actitud negativa y de indiferencia frente a los niños; no interactúan con ellos, no establecen contacto visual, no se evidencia preocupación o interés por mantener un acercamiento físico, ya sea para supervisar el trabajo efectuado por el estudiante u ofrecer algún apoyo acorde a sus características y necesidades educativas, impidiendo de esta manera el acceso a los contenidos curriculares. Por otra parte, también hay profesores que permanentemente interactúan con sus alumnos, ya sea a través del contacto visual, sonrisas y uso de refuerzo positivo, aspecto que incide positivamente en el interés del niño/a por participar de la clase. La actitud de los niños/ as integrados frente a la clase está supeditada a las formas predominantes de relación que se establezcan en el aula entre el profesorado y el estudiante, a la ubicación espacial del niño en la sala y las adaptaciones que realice el educador. Las representaciones que tienen los profesores con respecto a los niños/as con necesidades educativas especiales se relacionan con dificultades o problemas, destacándose la importancia del profesor especialista en el proceso de intervención pedagógica, lo cual deja entrever la persistencia del enfoque clínico rehabilitador, en el cual la atención diferenciada y la participación activa del educador especialista juega un rol importante. Los sentimientos que inspiran estos niños/as al profesorado son de cariño, afecto, protección, ayuda, pena, lástima, conmovedores e incluso les dan una connotación divina. Se infiere que este tipo de sentimiento podría incidir en el proceso de integración, dado que las expectativas de los docentes con respecto a estos niños podrían interferir en la práctica pedagógica, al tomar decisiones con respecto a la forma de responder a las necesidades educativas especiales a nivel de aula. La representación de los educadores respecto a la respuesta educativa para la atención de las necesidades educativas especiales en el aula, se manifiesta en el apoyo personalizado, las adaptaciones curriculares y en algunos casos se plantea el requerimiento de disponer de espacios especiales para los niños con discapacidad. Esto último deja entrever la persistencia de una mirada segregadora que excluye del sistema educativo a ciertos niños con capacidades diferentes.

Desde la perspectiva de las potencialidades, los profesores perciben a estos niños como poseedores de habilidades artísticas y sociales, destacando que éstos son más cariñosos que otros niños y además algunos docentes manifiestan que son también inteligentes. Desde la óptica de las limitaciones, el profesorado percibe a este estudiante disminuido intelectualmente, reflejado en un nivel de aprendizaje más lento, con dificultades para efectuar actividades de tipo individual y la tendencia a olvidar rápidamente aquello que se les enseña. Este tipo de convicción acerca de las capacidades intelectuales podría incidir notablemente en el proceso de integración escolar de los niños con discapacidad, dado que limitaría el acceso a las diferentes experiencias educativas y de conocimiento importante para el desenvolvimiento en la vida familiar, social y escolar. En general los educadores tienen un pensamiento positivo frente a la integración escolar, sin embargo, la intervención educativa que proponen para los niños con necesidades educativas especiales se encuentra cimentada bajo el modelo correspondiente al nivel D. (Hegarty,1981 citado en Marchesi 1998), esto es, tiempo compartido entre el aula de recursos y la sala común. Por otra parte, a pesar de estar de acuerdo con la integración de estos niños al aula común, aún prevalece la idea en parte del profesorado, de que la escuela especial constituye el espacio educativo más apropiado para ellos. Los sentimientos de competencia del profesorado frente a la integración escolar, deja de manifiesto la inseguridad que sienten al verse enfrentados a este proceso. Es percibida como una actividad compleja que genera incomodidad. Los factores que estarían incidiendo, de acuerdo a las opiniones vertidas, se vincularían a la falta de preparación para dar respuesta a las necesidades educativas especiales y a la falta de experiencia en el trabajo pedagógico con niños que poseen características distintas a las del común de los estudiantes. Los facilitadores que consideran prioritarios algunos de los docentes para que el proceso de integración pueda desarrollase adecuadamente, 
lo constituye el diagnóstico claro y oportuno de cada uno de los niños integrados al momento de hacer ingreso a la escuela. También destacan la importancia de educar en espacios distintos a los niños que presentan algún tipo de discapacidad con el objeto de que aprendan mejor. Desde esta perspectiva, se concluye que aún persiste la tendencia de considerar el modelo clínico rehabilitador como el más adecuado para responder a las características de este tipo de niños, como así también la existencia de actitudes segregacionistas. Otros elementos considerados importantes para la adecuada integración escolar es la capacitación, la existencia de material didáctico apropiado a las características de los niños, la participación de la familia, la actitud de los niños del curso y de los profesores de aula y la realización de adaptaciones curriculares. Aspectos que no se condicen en su mayoría en las actividades pedagógicas observadas. Los principales obstaculizadores para lograr un adecuado proceso de integración, según la opinión del profesorado, corresponderían al excesivo número de alumnos existente en cada curso y tener más de un niño integrado en el aula. Con respecto a los apoyos ofrecidos en el aula para responder a las necesidades educativas de los niños integrados, casi todos los profesores manifiestan hacer adaptaciones curriculares, otorgar atención personalizada, utilizar material atractivo y de interés para los niños, hacerlos partícipes de todas las actividades de la clase, elementos no presentes en la práctica pedagógica, evidenciándose una clara incongruencia entre lo que argumentan en la entrevista y su actuación pedagógica.

El rol de la escuela, según la percepción de los profesores, debe tender hacia la aceptación de los alumnos con necesidades educativas especiales, relevando la importancia que tiene la capacitación del profesorado, la gestión de espacios adecuados para atender a los niños, $y$ el establecimiento de ciertos tiempos para hacer reuniones que permitan el intercambio de experiencias pedagógicas en función de los apoyos requeridos por cada estudiante integrado. El rol de profesorado en el contexto de una escuela integradora es visualizado como importante en este proceso. La atención personalizada, el permanente apoyo, la motivación que se le ofrece al niño y las actividades desafiantes son indispensables para fomentar un autoestima positiva y el logro de aprendizaje por parte de los niños. Considerando distinciones de género, edad y años de servicio de los profesores que formaron parte del estudio, no se evidencian diferencias en las actitudes y representaciones que tienen con respecto a los niños con necesidades educativas especiales y el proceso de integración escolar.

Consideramos que una relación pedagógica debe ser flexible, no vertical, ni autoritaria, que se base en el intercambio dialógico de sujetos activos, es decir el estudiante no se debe considerar pasivo, el profesor debe facilitar un ambiente de construcción subjetiva para que ambos busquen apropiarse de un conocimiento, argumentando y controvirtiendo, lo cual solo se puede llevar a cabo con procesos comunicativos y respetuosos de interlocución e ideológicos.

\section{Recomendaciones}

Los resultados obtenidos, llevan a los autores a proponer algunas consideraciones que apuntan a la reflexión y discusión académica, necesaria para mejorar el proceso de enseñanza aprendizaje desde la mirada de una educación inclusiva, en la Institución Educativa María Inmaculada, en los casos analizados.

La educación inclusiva más que una política de estado debe convertirse en una oportunidad que posibilite tanto a docentes como directivos docentes, valorar a todos los estudiantes desde una perspectiva integradora, incluyente con procedimientos que permitan redireccionar en el docente su quehacer pedagógico y al estudiante su interés por mejorar los mecanismos de aprendizaje, haciéndolo consciente de sus dificultades, pero también de sus avances en el proceso formativo.

La institución debe propiciar espacios para implementar talleres y seminarios que favorezcan el aporte académico y la reflexión crítica sobre el quehacer pedagógico, en las diferentes disciplinas que se orientan. Con lo 
anterior, se puede motivar a la investigación y formación académica, donde los actores del proceso educativo compartan saberes y experiencias, aportando con ello a la consecución de estrategias pedagógicas incluyentes, para favorecer el aprendizaje de los estudiantes. Así mismo, estos espacios que implemente la institución, deben tener en cuenta al padre de familia como parte del proceso de enseñanza - aprendizaje de los estudiantes, para la socialización y retroalimentación de las políticas de inclusión.

Velar porque el ambiente de aplicación de las situaciones de enseñanza-aprendizaje sea similar en cuanto a recursos, espacios y tiempos. Además, se le debe aplicar al mismo estudiante variadas didácticas orientadas hacia los mismos aprendizajes. Así mismo, procurar que las dinámicas de aula incluyan contenidos más relevantes, para demostrar el logro del aprendizaje.

Tener en cuenta la planificación en la enseñanza, dado que ésta brinda elementos fundamentales para incluir a los estudiantes con barreras para el aprendizaje y reconoce los ambientes de aprendizaje requeridos para desarrollar el proceso de enseñanza- aprendizaje incluyente.

Por otra parte, a partir de los hallazgos se puede proponer la continuación de un ejercicio investigativo que trascienda más allá de reconocer las concepciones que tienen los docentes referentes al tema de educación inclusiva. Es decir, un trabajo de intervención en el proceso de enseñanza-aprendizaje incluyente, con ampliación a otros miembros de la comunidad educativa, docentes de todos los niveles, directivos docentes, padres de familia y estudiantes de los diferentes grados. Por lo anterior, la presente investigación se convierte en un antecedente para continuar en la institución con proyectos investigativos, que promuevan la implementación de estrategias para toda la población con barreras para el aprendizaje.

\section{Referencias Bibliográficas}

Álvarez, E. (2011). Perfil del docente inclusivo. Foro sobre Educación Inclusiva en Perú. Noviembre.

Ainscow, M.; Booth, T. \& Dyson, A. (2006). Improving schools, developing inclusion Londres: Routledge.

Ainscow, M. \& César, M. (2006). Inclusive Education ten years after Salamanca: Setting the agenda. Revista Europea de Psicología de la Educación XXI (3), 231-238.

Arnaiz, P. (2003). Educación inclusiva: una escuela para todos. Málaga: Aljibe.

Arnaiz, P. (2012). Escuelas eficaces e inclusivas cómo favorecer su desarrollo. Universidad de Murcia. Educatio Siglo XXI, Vol. 30 no 1 - 2012, pp. 25-44

Blanco, Rosa (2006) “La equidad y la inclusión social: uno de los desafíos de la escuela hoy, Ed. Revista Electrónica Iberoamericana sobre Calidad, Eficiencia y Cambio en Educación (REICE) Vol. 4. No. 3.

Blanco, Rosa (2007). “Los Docentes y el Desarrollo de escuelas Inclusivas. Revista Prelac.

Colombia. Datos de sobre educación. 6⿳a . Edición 2006/07.

Declaración Universal de los Derechos del Niño 20-11-1959

Díaz. H, O. y Franco. M, F. (2008). "Percepción y actitudes hacia la inclusión educativa de los docentes de Soledad, Atlántico (Colombia). Zona Próxima. Revista del Instituto de Estudios en Educación Universidad del Norte no 12 enero-junio, 2010 ISSN 1657-2416.

Domínguez, J. (2007). Una escuela democrática para una sociedad democrática. En J. Domínguez, y R. Feito, Finalidades de la educación en una sociedad democrática (pp. 7-128). Madrid: Octaedro 
Durán. D; y Giné. C. (2010). La formación del profesorado para la educación inclusiva: Un proceso de desarrollo profesional y de mejora de los centros para atender la diversidad. Revista Latino Americana de Educación Inclusiva.

Escarbajal, A. Mirete, A. Maquilón, J. Izquierdo, T. López, J. Orcajada, N; Sánchez, M. (2012). La atención a la diversidad: la educación inclusiva. Revista Electrónica Interuniversitaria de Formación del Profesorado REIFOP, 15 (1), 135-144. Recuperado en web: http//www.aufop. com.

Echeita, G. (2008). Inclusión y exclusión educativa. "voz y quebranto".

REICE, 2, (6), 9-18.

El Marco de acción de Dakar, (2000).

Foro Mundial de Educación para Todos (2000) Marco de Acción de Dakar de Educación para Todos: cumplir nuestros compromisos comunes. Dakar, Senegal, UNESCO, Paris.

Foro Consultivo Internacional sobre Educación para Todos (2000). Dakar, Senegal,

García, C, Vargas, O. (2012). Currículo inclusivo en contextos de vulnerabilidad escolar, Tesis de Maestría. Barranquilla- Colombia .

García, J. (2009). Aulas inclusivas. 13739 - Bordon 60-4 (FF).qxd 6/2/09 08:43 Página 89

Garzón, B. (2011). Reaccionar para avanzar. En Sampedro, J.L. y otros.

Reacciona (pp. 45-60). Madrid: Aguilar

Giordan, A (1995). Los orígenes del saber. De las concepciones personales a los conceptos científicos. Díada Editora S.L. Sevilla. Segunda Edición.

González Lara, Mireya. (2008). Paradojas en la formación docente. Elementos para avanzar en su reflexión y planteamiento de propuestas. Bogotá, D.C., Colombia.
Hargreaves, A (2003). Profesorado, Cultura y Postmodernidad: Cambian los tiempos, cambian el profesorado. Ediciones Morata S.L. Madrid- España

Infante, M. Desafíos a la formación docente: inclusión educativa Estudios Pedagógicos, vol. XXXVI, núm. 1, 2010, pp. 287-297, Universidad Austral de Chile.

López, M. (2009). La inclusión educativa de los alumnos con discapacidades graves $y$ permanentes en la Unión Europea. RELIEVE, v. 15, n. 1, 1-20.

Lopera, J. (2004). El maestro ante la diversidad. En: Modulo 1 de capacitación, Medellín, Diversidad y Educación.

Martínez, D (2011). Boletín especial de la Fundación Saldarriaga Concha sobre Educación Inclusiva Bogotá D.C., Colombia, abril de 2011.

Patiño Giraldo, Luz Elena. (2011). La atención a la diversidad en el contexto del aula de clase. Módulo Alternativas pedagógicas. Maestría Educación desde la Diversidad. Manizales: Universidad de Manizales.

Pozo, Juan Ignacio. (2006). Nuevas formas de pensar la enseñanza y el aprendizaje. Barcelona: Grao.

San Martín, C; Atención de la Diversidad en el contexto educativo Chileno: Concepciones del Profesorado sobre evaluación y diseño de la propuesta curricular Reice. Revista Iberoamericana sobre Calidad, Eficacia y Cambio en Educación, vol. 10, núm. 4, 2012, pp. 164 -183 Red Iberoamericana de Investigación Sobre Cambio y Eficacia Escolar Madrid, España.

Sandoval, M y Simón, C. (2007). "Estudios sobre las concepciones de la inclusión de los futuros maestros de la Universidad Autónoma de Madrid. 
Sapon-Shevin, M. (2012). La inclusión real: Una perspectiva de justicia social. Revista de Investigación en Educación, no 11 (3), 2013, pp. $71-85$

Trujillo, S. Torres, M. Leiton, F. Chilatra, F. 2010. Relaciones Pedagógicas. Epistemología de la Pedagogía. Licenciatura en Educación Básica con énfasis en Ciencias Sociales. Universidad de Amazonia. Florencia Caquetá.

UNESCO/OREALC (2000) Balance de los 20 años del Proyecto Principal de Educación para América Latina y el Caribe. UNESCO/ Santiago de Chile.

UNESCO (2004). Temario abierto sobre educación inclusiva. Santiago de Chile.

UNESCO (2005). Hacia las Sociedades del Conocimiento. Informe Mundial.

Valcárcel, M. (2010). De la Escuela Integradora a La Escuela Inclusiva. Facultad de Ciencias dela Educación de Ourense. Universidad de Vigo. Revista INNOVACIÓN EDUCATIVA, n..$^{\circ}$ 21, 2011: pp. 119-131

Verdugo, M. y Rodríguez, A. (2008). Valoración de la inclusión educativa desde diferentes perspectivas. Instituto Universitario de Integración en la Comunidad de la Universidad de Salamanca. Revista Española sobre Discapacidad Intelectual Vol 39 (3), Núm. 228, 2008 Pág. 5 a pág. 25.

Zaccagnini, Mario Cesar. (2008). Impacto de los paradigmas pedagógicos históricos en las prácticas educativas contemporáneas. Revista Iberoamérica de educación. 